\title{
MINIMIZERS WITH TOPOLOGICAL SINGULARITIES IN TWO DIMENSIONAL ELASTICITY*
}

\author{
JONATHAN BEVAN $^{1}$ AND XIAODONG YAN $^{2}$
}

\begin{abstract}
For a class of 2-D elastic energies we show that a radial equilibrium solution is the unique global minimizer in a subclass of all admissible maps. The boundary constraint is a double cover of $S^{1}$; the minimizer $u$ is $C^{1}$ and is such that $\operatorname{det} \nabla u$ vanishes at one point.
\end{abstract}

Mathematics Subject Classification. 49K15, 49K20, 49J30, 74B20.

Received October 28, 2005. Revised March 31, 2006.

Published online September 21, 2007.

\section{IntroduCtion}

In this short note we present a radial equilibrium solution $u$ for a class of variational integrals in two dimensional elasticity such that $u$ is the unique global minimizer in a subclass of admissible maps. The variational integrals we are interested in are of the form

$$
I(u)=\int_{\Omega} \gamma(\nabla u(x)) \mathrm{d} x
$$

where $\Omega \subset \mathbb{R}^{2}$ is a bounded domain, $u \in W^{1,2}\left(\Omega, \mathbb{R}^{2}\right)$, and $\gamma: M^{2 \times 2} \rightarrow[0, \infty]$ is a polyconvex function defined by

$$
\gamma(P)= \begin{cases}\frac{|P|^{2}}{2}+H(\operatorname{det} P) & \text { for } P \in M_{+}^{2 \times 2}, \\ +\infty & \text { for } P \in M^{2 \times 2}-M_{+}^{2 \times 2} .\end{cases}
$$

$H$ is a nonnegative convex function on $(0, \infty)$ and is proportional to $d^{-s}$ for all sufficiently small positive values of $d$ for some $s>0$. Here and throughout the paper we use $M^{2 \times 2}$ to denote the set of all $2 \times 2$ matrices and $M_{+}^{2 \times 2}$ denotes the subset of matrices in $M^{2 \times 2}$ whose determinant is positive.

Integrals of this type model the stored energy of certain nonlinearly elastic, rubber-like materials (see [2,13]). We are interested in the minimization of $I$ under a pure displacement boundary constraint. More precisely, we

Keywords and phrases. Nonlinear elasticity, singular minimizer, stability.

* We thank John Ball for many helpful discussions. J. Bevan's research is supported by an EPSRC Postdoctoral Research Fellowship. X. Yan's research is partially supported by a Research Challenge Fund from New York University and NSF Grant DMS-0431710. X. Yan's research is also supported by an IRGP grant from Michigan State University. We thank two referee for many helpful suggestions. We also thank Stefan Müller for his comments on an earlier version of this paper.

1 Department of Mathematics, University of Surrey, Guildford, GU2 7XH, UK; j.bevan@surrey.ac.uk

2 Department of Mathematics, Michigan State University, East Lansing, MI 48824, USA; xiayan@math.msu.edu

(c) EDP Sciences, SMAI 2007 
minimize $I(u)$ on the set

$$
\mathcal{A}_{g}=\left\{u \in W^{1,2}\left(\Omega, \mathbb{R}^{2}\right): \operatorname{det} \nabla u>0 \text { a.e. in } \Omega, u=g \text { on } \partial \Omega\right\}
$$

where $g$ is the double-covering map

$$
g(R, \theta)=(R, 2 \theta)
$$

and $\Omega$ is the unit ball in $\mathbb{R}^{2}$. Under suitable conditions concerning the quasiconvexity, differentiability and growth of $\gamma$, it can be shown $[1,6]$ that there exists at least one minimizer in $\mathcal{A}_{g}$ whenever $\mathcal{A}_{g}$ is nonempty. In general, it is not known if a minimizer attains higher regularity or whether it is unique in $\mathcal{A}_{g}$.

Properties of minimizers are closely related to properties of stationary states. There are two types of stationary state associated with the energy $I$. Consider variations of the form $u_{\epsilon}(x)=u(x)+\epsilon \phi(x)$ for $\phi \in C_{0}^{1}\left(\Omega, \mathbb{R}^{2}\right)$; the first variation at $\epsilon=0$ formally gives the Euler-Lagrange equations corresponding to $I$

$$
\left(\frac{\partial \gamma}{\partial P_{\alpha}^{k}}(\nabla u)\right)_{x_{\alpha}}=0 \quad \text { in } \mathcal{D}^{\prime}(\Omega)
$$

for $k=1,2$. A second class of variations are of the form $u_{\epsilon}(x)=u(x+\epsilon \phi(x))$ for $\phi \in C_{0}^{1}\left(\Omega, \mathbb{R}^{2}\right)$; the first variation at $\epsilon=0$ gives the equilibrium equations corresponding to $I$

$$
\left[-\gamma(\nabla u) \cdot \delta_{\alpha}^{k}+u_{x_{k}}^{i} \cdot \frac{\partial \gamma}{\partial P_{\alpha}^{i}}(\nabla u)\right]_{x_{\alpha}}=0 \text { in } \mathcal{D}^{\prime}(\Omega)
$$

for $k=1,2$.

Interesting work on the regularity and uniqueness of weak solutions to (1.3) and (1.4) can be found in $[7,8,11,20]$. Due to the nonconvexity of $I$ and the singular behaviour of $\gamma(P)$ near $\operatorname{det} P=0$ it is not known whether a minimizer (which necessarily satisfies (1.4)) satisfies (1.3). Conversely, it is quite difficult to determine when a weak solution of the equilibrium equations or Euler-Lagrange equations corresponding to an elastic energy functional is a local or global minimizer. Using ideas from the field theory of the calculus of variations, Sivaloganathan [15] developed a general technique for demonstrating the stability of $C^{2}$ solutions of the equilibrium equations (equivalent to the Euler-Lagrange equations when the solution is $C^{2}$ ) for a large class of polyconvex functionals in finite elasticity. In a similar spirit, Zhang [21] later proved stability for any $C^{1}$ weak solution $u$ of the Euler-Lagrange equations with det $\nabla u(x)>0$ everywhere. In this context stability means that $u$ is a local minimizer in $W^{1, p}$ with respect to variations $u+\varphi$ where the support of $\varphi$ is sufficiently small. The method employed in [15] and [21] is a direct argument based on the theory of the Null Lagrangian (see e.g. [2,5]). Another successful application of the method is by Zhang [22] who, for a pure boundary displacement problem in three dimensions with small boundary displacement from a stress-free state, shows that the smooth solution of the equilibrium equations given by the implicit function theorem is the unique global minimizer. The same argument also extends to boundary conditions which are small pertubations of a linear map. As Zhang remarks in [22], this seems to be the only situation in which the smoothness of the global minimizer has been proved. Special cases of this type can also be found in [14].

The stability of special discontinuous solutions to the equilibrium equations, such as those which exhibit the cavitation phenomenon studied by Ball [3], has attracted a lot of attention in the literature. For a class of isotropic stored energy functions of slow growth $(p<n)$, Ball analyzed the radial problem on the unit ball in $\mathbb{R}^{n}(n \geq 3)$ subject to the boundary constraint $u_{\lambda}: x \mapsto \lambda x$. He showed that there is a critial value $\lambda_{\mathrm{c}}$ such that for $\lambda<\lambda_{\mathrm{c}}, u_{\lambda}$ is a global minimizer among all radial mappings, while for $\lambda>\lambda_{\mathrm{c}}$ the radial solution $u(x)=r(|x|) \frac{x}{|x|}$ with $r(0)>0$ has lower energy and is a global minimizer among all radial mappings. Following Ball's work, a number of further results on cavitation were obtained (see the survey paper [10] and the references therein). Most work concentrated on the radial problem. A question which remains unanswered in this area is the following: are the cavitating solutions obtained by Ball and others global or local minimizers? Partial results can be found in [16-18]. In particular, for a large class of materials, cavitating minimizers are local 
minimizers with respect to small and continuous perturbations which are supported away from the cavity; and for a special stored energy functional

$$
\gamma(F)=a|F|^{2}+b \operatorname{det} F
$$

$(a>0, b>0)$, Sivaloganathan [16] showed that the radial cavitating minimizer is indeed the global minimizer among deformations which only create a single hole at the centre of the ball. The proof, however, depends crucially on the special structure of the stored energy functional (1.5).

For two-dimensional models in the form of (1.1), (1.2), cavitation never appears in weak solutions to the equilibrium equations with finite energy (see e.g. [19]). In fact, under linear boundary constraints a direct argument using Null Lagrangians shows that the linear map is the unique global minimizer. It is therefore interesting to look at stability problems for nonsmooth solutions to (1.3) or (1.4) subject to nonlinear boundary constraints.

In their study of the maximal smoothness of stationary solutions $u$ for functionals of the form (1.1) and (1.2), Bauman, Owen and Phillips [8] proved that any $C^{1, \alpha}$ solution to (1.4) is smooth and satisfies det $\nabla u>0$ everywhere in the domain. In particular, this implies the stability of any $C^{1, \alpha}$ solution by the results of Sivalogonathan and Zhang [15,21]. In the same paper, Bauman et al. considered weak solutions to (1.4) subject to the double-covering boundary condition. They obtained a global minimizer $u_{0}$ among all radial mappings having a certain double-twist structure which is also a weak solution of both (1.3) and (1.4). The solution $u_{0}$ is $C^{1}$ and it satisfies $\operatorname{det} \nabla u_{0}(x)>0$ everywhere in the domain apart from the origin where $\operatorname{det} \nabla u_{0}(0)=0$. It then follows from Zhang's result [21] that $u_{0}$ is stable subject to any perturbation with sufficiently small support away from the origin. It is not known, however, whether $u_{0}$ is a local or a global minimizer in any class which strictly contains the class $\mathcal{A}_{s}$ of 'double-twist' maps that Bauman et al. considered in their original work (see (2.2) for the definition of $\mathcal{A}_{s}$ ). We remark that the properties of $u_{0}$ described in [8] do not depend on its being a minimizer in $\mathcal{A}_{s}$ but on its being a solution of the equilibrium equations (1.4). Thus a simple consequence of our Theorem 1 is that $u_{0}$ is the unique solution of the equilibrium equations in the class $\mathcal{A}_{s}$ (see Corr. 1). The weaker conclusion that $u_{0}$ is the unique minimizer in $\mathcal{A}_{s}$ is a simple consequence of Theorem 1 .

Our main purpose in this paper is to study the stability of the radial solution $u_{0}$ constructed in [8] by Bauman, Owen and Phillips. We show that $u_{0}$ is the unique global minimizer in a subclass $\mathcal{A}^{-}$of all admissible maps. The subclass $\mathcal{A}^{-}$is defined in (2.5); for the moment we note that it strictly includes all admissible maps with Fourier modes higher than or equal to 2 . Although we focus on a particular case, our results are stronger than those of Sivaloganathan [15] and Zhang [21] in the sense that $I\left(u_{0}+\varphi\right)>I\left(u_{0}\right)$ for any admissible variation $u_{0}+\varphi \in \mathcal{A}^{-}, \varphi \neq 0$, regardless of the size of the support of $\varphi$.

The main ingredients in our proof are new estimates of $\operatorname{det} \nabla u_{0}$ near the origin, the special structure of $\operatorname{det} \nabla u_{0}$ which depends only on the radial direction, the fact that $u_{0}$ satisfies the Euler-Lagrange equation (1.3), and a trick to rewrite $\operatorname{det} \nabla \varphi$ as $\operatorname{Det} \nabla \varphi$ for smooth functions. It turns out that these estimates, together with an approximation argument based on the duality $\left(\mathcal{H}^{1}\right)^{*}=B M O$ and a result of Coifman et al. [9], are sufficient to prove that $u_{0}$ is indeed the unique global minimizer in $\mathcal{A}^{-}$.

We first briefly review some facts in Section 2 and in Section 3 we present the proof of our main results. In the last section we state some open problems for future work.

\section{Preliminaries}

Let $\mathbb{B}_{1} \subset \mathbb{R}^{2}$ be the unit disk. We consider variational integrals of the form

$$
I(u)=\int_{\mathbb{B}_{1}} \gamma(\nabla u(x)) \mathrm{d} x,
$$


where $u \in W^{1,2}\left(\mathbb{B}_{1}, \mathbb{R}^{2}\right), \gamma: M^{2 \times 2} \rightarrow[0, \infty]$ is defined by

$$
\gamma(P)=\left\{\begin{array}{ccc}
\frac{|P|^{2}}{2}+H(\operatorname{det} P) & \text { for } & P \in M_{+}^{2 \times 2} \\
+\infty & \text { for } & P \in M^{2 \times 2}-M_{+}^{2 \times 2}
\end{array}\right.
$$

Here $H$ satisfies:

(1) $H$ is convex and $H \geq 0$ on $(0, \infty)$.

(2) $H \in C^{3}((0, \infty))$ and for some positive constants $s, c_{1}, c_{2}$, and $d_{0}$,

$$
c_{1} t^{-s-k} \leq(-1)^{k} \cdot \frac{\mathrm{d}^{k} H(t)}{\mathrm{d} t^{k}} \leq c_{2} t^{-s-k}
$$

for $0<t<d_{0}$ and $k=0,1,2$.

(3) $H(t)=+\infty$ for $t \leq 0$.

(4) For some real number $\tau$ and positive constants $c_{3}, c_{4}$ and $d_{1}$,

$$
c_{3} t^{\tau} \leq \frac{\mathrm{d}^{2} H(t)}{\mathrm{d} t^{2}} \leq c_{4} t^{\tau} \text { for } t \geq d_{1}
$$

Let

$$
\mathcal{A}=\left\{u \in W^{1,2}\left(\mathbb{B}_{1}, \mathbb{R}^{2}\right): I(u)<+\infty, \operatorname{det} \nabla u>0 \text { a.e. in } \mathbb{B}_{1},\left.u\right|_{\partial \mathbb{B}_{1}}=(1,2 \theta)\right\} .
$$

Consider the mapping $v: \mathbb{B}_{1} \rightarrow \mathbb{R}^{2}$ of the form

$$
v(x)=(s(R) \cdot \cos 2 \theta, s(R) \cdot \sin 2 \theta)
$$

with $x=(R \cos \theta, R \sin \theta)$ and set

$$
\mathcal{A}_{s}=\{v \in \mathcal{A}: v \text { is of the form (2.1) with } s(1)=1\} .
$$

Let $u_{0}$ denote a minimizer in $\mathcal{A}_{s}$. Baumann, Owen and Phillips [8] proved that

$$
u_{0}:(R, \theta) \rightarrow(r(R), 2 \theta)
$$

where $r$ satisfies the following properties:

(i) $r \in C^{1}([0,1]) \cap C^{3}((0,1])$;

(ii) $r(0)=0$ and $r(1)=1$;

(iii) $r^{\prime}(R)>0$ for $0<R<1$ and $r^{\prime}(0)=0$;

(iv) $r(R)$ satisfies in $\mathcal{D}^{\prime}((0,1))$

$$
\left(\frac{\left(r^{\prime}(R)\right)^{2}}{2}+f\left(2 \frac{r^{\prime}(R) \cdot r(R)}{R}\right)\right)^{\prime}=4 \frac{r^{\prime}(R) \cdot r(R)}{R^{2}}-\frac{\left(r^{\prime}(R)\right)^{2}}{R}
$$

where $f(d)=d H^{\prime}(d)-H(d)$.

(v) There exists $\delta_{0}>0$ such that $d=d(R)$ is monotone on $\left(0, \delta_{0}\right)$. Moreover, on $\left(0, \delta_{0}\right), d^{\prime}(R) \geq 0$ and $0<4-2 \sqrt{3} \leq \frac{R \cdot r^{\prime}(R)}{r} \leq 4+2 \sqrt{3}, \lim _{R \rightarrow 0^{+}} d(R)=0$. Here,

$$
d(R):=\operatorname{det} \nabla u_{0}=2 \frac{r^{\prime}(R) \cdot r(R)}{R} .
$$


(vi) $u_{0}$ satisfies the equilibrium equation and the Euler-Lagrange equation in $\mathcal{D}^{\prime}\left(\mathbb{B}_{1}\right)$, i.e. both

$$
\begin{gathered}
{\left[-\gamma(\nabla u) \cdot \delta_{\alpha}^{k}+u_{x_{k}}^{i} \cdot \frac{\partial \gamma}{\partial P_{\alpha}^{i}}(\nabla u)\right]_{x_{\alpha}}=0, \text { and }} \\
\left(\frac{\partial \gamma}{\partial P_{\alpha}^{k}}\left(\nabla u_{\mathcal{o}}\right)\right)_{x_{\alpha}}=0
\end{gathered}
$$

hold in $\mathcal{D}^{\prime}\left(\mathbb{B}_{1}\right)$ for $k=1,2$.

In the next section we show that $u_{0}$ is the unique global minimizer in a subclass of $\mathcal{A}$. We write the map $v \in \mathcal{A}$ as $v=u_{0}+\phi$, where

$$
\phi=\left(\phi_{1}, \phi_{2}\right) \in W_{0}^{1,2}\left(\mathbb{B}_{1}, \mathbb{R}^{2}\right)
$$

with

$$
\begin{aligned}
& \phi_{1}(R, \theta)=a_{0}(R)+a_{1}(R) \cos \theta+b_{1}(R) \sin \theta+\sum_{n \geq 2}^{\infty}\left(a_{n}(R) \cos n \theta+b_{n}(R) \sin n \theta\right) \\
& \phi_{2}(R, \theta)=c_{0}(R)+c_{1}(R) \cos \theta+d_{1}(R) \sin \theta+\sum_{n \geq 2}^{\infty}\left(c_{n}(R) \cos n \theta+d_{n}(R) \sin n \theta\right) .
\end{aligned}
$$

We shall concentrate on the following subset of $\mathcal{A}$ :

$$
\mathcal{A}^{-}=\left\{v \in \mathcal{A}: \int_{0}^{1} H^{\prime \prime}(d(R)) d^{\prime}(R)\left(a_{1}(R) d_{1}(R)-c_{1}(R) b_{1}(R)\right) \mathrm{d} R \leq 0\right\} .
$$

The integral in (2.5) is well-defined in spite of the singular behaviour of the function $H$ near the origin: this is a consequence of Lemmas 2 and 3 which follow in the next section. We are now in a position to state the main result of this paper.

Theorem 1. The Bauman-Owen-Phillips map $u_{0}$ is the unique global minimizer of $I$ in $\mathcal{A}^{-}$.

\section{Proof of main Results}

We prove Theorem 1 in several steps. First we prove the following growth estimates on $d(R)$ near $R=0$.

Lemma 1. With $r, d$ and $H$ as above,

(i) $\lim _{R \rightarrow 0^{+}} \frac{r^{\prime}(R) R}{r(R)}=1$;

(ii) $\lim _{R \rightarrow 0^{+}} R H^{\prime \prime}(d) d^{\prime}(R)=\frac{3}{2}$;

(iii) $\lim _{R \rightarrow 0^{+}} \frac{H^{\prime}(d(R))}{\ln R}=\frac{3}{2}$.

Proof. Recall that $r \in C^{1}([0,1]) \cap C^{3}((0,1])$ and satisfies in $\mathcal{D}^{\prime}((0,1))$

$$
\left(\frac{\left[r^{\prime}(R)\right]^{2}}{2}+f\left(2 \frac{r^{\prime}(R) \cdot r(R)}{R}\right)\right)^{\prime}=4 \frac{r^{\prime}(R) \cdot r(R)}{R^{2}}-\frac{\left[r^{\prime}(R)\right]^{2}}{R}
$$

that is,

$$
r^{\prime}\left(r^{\prime \prime}+\frac{r^{\prime}}{R}-4 \frac{r}{R^{2}}\right)+f^{\prime}(d) d^{\prime}=0 \text { for } R \in(0,1)
$$


From assumption (2) on $H$ and (v), we have $f^{\prime}(d)=d H^{\prime \prime}(d)>0$ and $d^{\prime}(R) \geq 0$ on $\left(0, \delta_{0}\right)$, therefore equation $(3.1)$ implies

$$
\begin{aligned}
& r^{\prime}\left(r^{\prime \prime}+\frac{r^{\prime}}{R}-4 \frac{r}{R^{2}}\right) \leq 0 \quad \text { for } 0<R<\delta_{0}, \\
& d^{\prime}(R)=2\left(\frac{r^{\prime \prime} r}{R}+\frac{r^{\prime 2}}{R}-\frac{r^{\prime} r}{R^{2}}\right) \geq 0 \text { for } 0<R<\delta_{0}
\end{aligned}
$$

Now (3.2) can be rewritten as

$$
\begin{gathered}
r^{\prime \prime} R+r^{\prime}-4 \frac{r}{R} \leq 0 \quad \text { for } \quad 0<R<\delta_{0}, \\
r^{\prime \prime} R+\frac{r^{\prime 2} R}{r}-r^{\prime} \geq 0 \quad \text { for } \quad 0<R<\delta_{0} .
\end{gathered}
$$

Letting $R \rightarrow 0^{+}$in (3.3) and taking into account that $r^{\prime}(R) \rightarrow 0$ and $r^{\prime}(R)$ is proportional to $\frac{r(R)}{R}$ we conclude from (3.3) that

$$
r^{\prime \prime}(R) R \rightarrow 0
$$

On the other hand, (3.1) can be rewritten as

$$
r^{\prime}\left(r^{\prime \prime}+\frac{r^{\prime}}{R}-4 \frac{r}{R^{2}}\right)+2 d H^{\prime \prime}(d)\left(\frac{r^{\prime \prime} r}{R}+\frac{r^{\prime 2}}{R}-\frac{r^{\prime} r}{R^{2}}\right)=0
$$

Substituting (3.4) into (3.5) we have

$$
d H^{\prime \prime}(d) R\left(\frac{r^{\prime \prime} r}{R}+\frac{r^{\prime 2}}{R}-\frac{r^{\prime} r}{R^{2}}\right) \rightarrow 0 \text { as } R \rightarrow 0^{+}
$$

In particular, this implies

$$
\frac{1}{2} \mathrm{~d}^{2} H^{\prime \prime}(d)\left(\frac{r^{\prime \prime} R}{r^{\prime}}+\frac{r^{\prime} R}{r}-1\right) \rightarrow 0 \text { as } R \rightarrow 0^{+} .
$$

Assumption (2) on $H$ and (3.6) imply that

$$
\frac{r^{\prime \prime} R}{r^{\prime}}+\frac{r^{\prime} R}{r}-1 \rightarrow 0 \text { as } R \rightarrow 0^{+}
$$

which is equivalent to

$$
\frac{r^{\prime \prime} r+r^{\prime 2}}{\frac{r^{\prime} r}{R}} \rightarrow 1 \text { as } R \rightarrow 0^{+}
$$

By l'Hôpital's Rule, (3.8) implies

$$
\frac{r^{\prime} r}{\int_{0}^{R} \frac{r^{\prime}(s) r(s)}{s} \mathrm{~d} s} \rightarrow 1 \text { as } R \rightarrow 0^{+}
$$


Applying l'Hôpital's Rule and (3.9) repeatedly we get

$$
\begin{aligned}
\lim _{R \rightarrow 0^{+}} \frac{r^{\prime} R}{r} & =\lim _{R \rightarrow 0^{+}} \frac{r^{\prime} r R}{r^{2}} \\
& =\lim _{R \rightarrow 0^{+}} \frac{R \int_{0}^{R} \frac{r^{\prime}(s) r(s)}{s} \mathrm{~d} s}{r^{2}} \lim _{R \rightarrow 0^{+}} \frac{r^{\prime} r}{\int_{0}^{R} \frac{r^{\prime}(s) r(s)}{s} \mathrm{~d} s} \\
& =\lim _{R \rightarrow 0^{+}} \frac{\int_{0}^{R} \frac{r^{\prime}(s) r(s)}{s} \mathrm{~d} s+r r^{\prime}}{2 r r^{\prime}} \\
& =1,
\end{aligned}
$$

proving part (i) of the lemma. To prove parts (ii) and (iii) note that (3.7) and (3.10) together imply

$$
\frac{r^{\prime \prime} R}{r^{\prime}} \rightarrow 0 \text { as } R \rightarrow 0^{+}
$$

Rewriting (3.5) in the form

$$
\frac{r^{\prime \prime} R}{r^{\prime}}+1-4 \frac{r}{R r^{\prime}}+\frac{R}{r^{\prime 2}} d H^{\prime \prime}(d) d^{\prime}=0
$$

and using the limits computed in (3.10) and (3.11) above we obtain

$$
\frac{R}{r^{\prime 2}} d H^{\prime \prime}(d) d^{\prime} \rightarrow 3 \text { as } R \rightarrow 0^{+} .
$$

Therefore

$$
\begin{aligned}
\lim _{R \rightarrow 0^{+}} R H^{\prime \prime}(d) d^{\prime} & =\lim _{R \rightarrow 0^{+}} \frac{R}{r^{\prime 2}} d H^{\prime \prime}(d) d^{\prime} \lim _{R \rightarrow 0^{+}} \frac{r^{\prime 2}}{d} \\
& =\frac{3}{2}
\end{aligned}
$$

and hence (ii) follows. Part (iii) follows from (ii) and l'Hôpital's Rule.

From the growth estimate (iii) above and the fact that $u_{0}$ is $C^{1}\left(\mathbb{B}_{1}\right), r^{\prime}(R)>0$ for $0<R<1$ and $r^{\prime}(0)=0$, we can easily conclude that $\frac{\partial \gamma\left(\nabla u_{0}\right)}{\partial P} \in L^{2}\left(\mathbb{B}_{1}\right)$. It then follows from $(2.4)$ through an approximation argument that $u_{0}$ is a weak solution of the Euler-Lagrange equation in the sense that

$$
\int_{\mathbb{B}_{1}} \frac{\partial \gamma\left(\nabla u_{0}\right)}{\partial P_{\alpha}^{k}} \cdot \psi_{x_{\alpha}}^{k} \mathrm{~d} x=0 \quad \text { for all } \psi \text { in } W_{0}^{1,2}\left(\mathbb{B}_{1}, \mathbb{R}^{2}\right)
$$

Lemma 2. With $d$ as above we have $d^{\prime}(R)>0$ for $R \in(0,1)$.

Proof. We first show $d^{\prime}$ can never vanish on $(0,1)$. Suppose for a contradiction that there existed $R_{0} \in(0,1)$ such that $d^{\prime}\left(R_{0}\right)=0$. Then we must have

$$
d^{\prime}\left(R_{0}\right)=\frac{2 r\left(R_{0}\right) r^{\prime \prime}\left(R_{0}\right)}{R_{0}}+2 \frac{\left(r^{\prime}\left(R_{0}\right)\right)^{2}}{R_{0}}-\frac{2 r\left(R_{0}\right) r^{\prime}\left(R_{0}\right)}{R_{0}^{2}}=0 .
$$

On the other hand, since $u_{0}=(r \cos 2 \theta, r \sin 2 \theta)$ satisfies the Euler-Lagrange equation with $r \in C^{3}((0,1])$, we have for $R \in(0,1)$,

$$
r^{\prime} r^{\prime \prime}+d H^{\prime \prime}(d) d^{\prime}-\frac{4 r r^{\prime}}{R^{2}}+\frac{r^{2}}{R}=0
$$


Since $r^{\prime}(R)>0$ in $(0,1),(3.15)$ can be rewritten as

$$
\frac{R_{0} r^{\prime \prime}\left(R_{0}\right)}{r^{\prime}\left(R_{0}\right)}+\frac{R_{0} r^{\prime}\left(R_{0}\right)}{r\left(R_{0}\right)}-1=0
$$

while $d^{\prime}\left(R_{0}\right)=0$ in $(3.16)$ gives

$$
\frac{R_{0}^{2} r^{\prime \prime}\left(R_{0}\right)}{r\left(R_{0}\right)}+\frac{R_{0} r^{\prime}\left(R_{0}\right)}{r\left(R_{0}\right)}-4=0
$$

Subtracting (3.17) from (3.18) gives

$$
\frac{R_{0}^{2} r^{\prime \prime}\left(R_{0}\right)}{r\left(R_{0}\right)}>\frac{R_{0} r^{\prime \prime}\left(R_{0}\right)}{r^{\prime}\left(R_{0}\right)},
$$

which, if $r^{\prime \prime}\left(R_{0}\right)>0,(3.19)$ implies

$$
\frac{R_{0}}{r\left(R_{0}\right)}>\frac{1}{r^{\prime}\left(R_{0}\right)}
$$

contradicting (3.17). If on the other hand $r^{\prime \prime}\left(R_{0}\right)<0$ then $(3.19)$ implies

$$
\frac{R_{0}}{r\left(R_{0}\right)}<\frac{1}{r^{\prime}\left(R_{0}\right)}
$$

which also contradicts (3.17). Thus $d^{\prime}(R)$ never changes sign in $(0,1)$. From [8], Lemma 3.7 and Corollary 3.8 , we know $d \in C^{2}((0,1))$ and

$$
d^{\prime}(R)>0 \text { near } R=0
$$

so that we must have $d^{\prime}(R)>0$ on $(0,1)$.

Assumption (1) on $H$ and Lemma 2 implies $g(R):=R H^{\prime \prime}(d(R)) d^{\prime}(R) \geq 0$ on $(0,1)$. In the next lemma we prove an upper bound on $g(R)$.

Lemma 3. With $g$ as above we have $g(R) \leq 2$ on $(0,1]$.

Proof. By the Euler-Lagrange equation (3.1), $g(R)$ may be written as

$$
g(R)=2-\frac{R r^{\prime}}{2 r}-\frac{R^{2} r^{\prime \prime}}{2 r}
$$

for each $0<R \leq 1$. The function $g$ can be made right-continuous at 0 by removing the singularity and defining $g(0):=\frac{3}{2}$, the limit $\lim _{R \rightarrow 0^{+}} g(R)$ having already been computed in (3.13). Thus $g(R)<2$ if $R$ is sufficiently small. Moreover, since $g$ is left-continuous at 1 we need only show that $g(R)<2$ for each $R \in(0,1)$. We now fix $R_{1}$ in $(0,1)$ and show that $g\left(R_{1}\right)<2$. There are two cases according to whether $r^{\prime \prime}\left(R_{1}\right) \geq 0$ or $r^{\prime \prime}\left(R_{1}\right)<0$.

Case I: $r^{\prime \prime}\left(R_{1}\right) \geq 0$. By equation (3.20) and the fact that $r^{\prime}(R)>0$ on $(0,1)$ we immediately have $g\left(R_{1}\right)<2$.

Case II: $r^{\prime \prime}\left(R_{1}\right)<0$. We claim that there exists $R_{0}<R_{1}$ such that $r^{\prime \prime}\left(R_{0}\right)=0$, for otherwise $r^{\prime \prime}(s)<0$ for all $s \in\left(0, R_{1}\right)$ which implies in particular that $r^{\prime}\left(R_{1}\right)<0$, a contradiction. Let $R_{0}=\max \left\{R \in\left(0, R_{1}\right]: r^{\prime \prime}(R)=0\right\}$. The preceding argument shows that $R_{0}>0$ and that $r^{\prime \prime}$ is strictly negative on the interval $\left(R_{0}, R_{1}\right)$. Lemma 2 tells us that $d^{\prime}(R)>0$ on $(0,1)$, that is,

$$
\frac{r^{\prime \prime}(R) R}{r^{\prime}(R)}+\frac{r^{\prime}(R) R}{r(R)}-1>0 .
$$

Hence

$$
\frac{r^{\prime}(R) R}{r(R)}>1 \text { on }\left(R_{0}, R_{1}\right)
$$


the inequality being strict because $\frac{r^{\prime \prime}(R) R}{r^{\prime}(R)}<0$ on $\left(R_{0}, R_{1}\right)$. Let $h(R)=\frac{r^{\prime}(R) R}{r(R)}$ for $R>0$. We have

$$
h^{\prime}(R)=\frac{r^{\prime}}{r}\left(\frac{R r^{\prime \prime}}{r^{\prime}}+1-h(R)\right)
$$

which in view of $(3.22)$ and $\left.r^{\prime \prime}\right|_{\left(R_{0}, R_{1}\right)}<0$ gives $h^{\prime}(R)<0$ for $R_{0}<R<R_{1}$. Hence $h\left(R_{0}\right)>1$, since otherwise for any $R \in\left(R_{0}, R_{1}\right)$ we would have $h(R)<h\left(R_{0}\right) \leq 1$, contradicting (3.22). By part (i) of Lemma 1 we know $\lim _{R \rightarrow 0^{+}} h(R)=1$, which, since $h\left(R_{0}\right)>1$ and $\left.h^{\prime}\right|_{\left(R_{0}, R_{1}\right)}<0$, means that $h$ has a maximum point at some $c \in\left(0, R_{1}\right)$. In particular we have $h(R) \leq h(c)$ for all $0<R<R_{1}$. Since $h^{\prime}(c)=0$, we have

$$
c r^{\prime \prime}(c)=r^{\prime}(c)(h(c)-1),
$$

and hence from (3.20)

$$
\begin{aligned}
g(c) & =2-\frac{h(c)}{2}-\frac{h(c)}{2}(h(c)-1) \\
& =2-\frac{(h(c))^{2}}{2} .
\end{aligned}
$$

Since $g(R)>0$ on $(0,1)$ it follows that $h(c) \leq 2$. Evaluating $g$ at $R_{1}$ and using $(3.21)$ we have

$$
\begin{aligned}
g\left(R_{1}\right) & =2-\frac{h\left(R_{1}\right)}{2}-\frac{R_{1}}{2 r\left(R_{1}\right)} R_{1} r^{\prime \prime}\left(R_{1}\right) \\
& \leq 2-\frac{h\left(R_{1}\right)}{2}+\frac{R_{1}}{2 r\left(R_{1}\right)}\left(h\left(R_{1}\right)-1\right) r^{\prime}\left(R_{1}\right) \\
& =2-h\left(R_{1}\right)+\frac{h^{2}\left(R_{1}\right)}{2} \\
& <2,
\end{aligned}
$$

where the last inequality follows from $h\left(R_{1}\right)<h(c) \leq 2$. This finishes the proof of the lemma.

The groundwork for the proof of Theorem 1 is almost complete. It remains for us to compare the energies of $u_{0}$ and $u_{0}+\varphi \in \mathcal{A}^{-}$. Since $H$ is a convex function we have

$$
\begin{aligned}
\gamma\left(\nabla u_{0}+\nabla \varphi\right) & =\frac{1}{2}\left|\nabla u_{0}+\nabla \varphi\right|^{2}+H\left(\operatorname{det}\left(\nabla u_{0}+\nabla \varphi\right)\right) \\
& \geq \frac{1}{2}\left|\nabla u_{0}\right|^{2}+\nabla u_{0} \cdot \nabla \varphi+\frac{1}{2}|\nabla \varphi|^{2}+H\left(\operatorname{det} \nabla u_{0}\right) \\
& +H^{\prime}\left(\operatorname{det} \nabla u_{0}\right)\left(\operatorname{det}\left(\nabla u_{0}+\nabla \varphi\right)-\operatorname{det} \nabla u_{0}\right) .
\end{aligned}
$$

This, together with the fact that $u_{0}$ is a weak solution of (3.14) implies that

$$
I\left(u_{0}+\varphi\right) \geq I\left(u_{0}\right)+J\left(u_{0}, \varphi\right),
$$

where

$$
J\left(u_{0}, \varphi\right)=\int_{\mathbb{B}_{1}} \frac{1}{2}|\nabla \varphi|^{2}+H^{\prime}\left(\operatorname{det} \nabla u_{0}\right) \operatorname{det} \nabla \varphi \mathrm{d} x
$$

The force of Proposition 1 below is that

$$
J\left(u_{0}, \varphi\right) \geq 0
$$


if $u_{0}+\varphi \in \mathcal{A}^{-}$. This is proved first for smooth $\varphi$ and then by an approximation argument for any $\varphi$ in $W_{0}^{1,2}\left(\mathbb{B}_{1}, \mathbb{R}^{2}\right)$ such that $u_{0}+\varphi \in \mathcal{A}^{-}$. We shall see that $J\left(u_{0}, \cdot\right)$ is continuous with respect to strong convergence in $W_{0}^{1,2}\left(\mathbb{B}_{1}, \mathbb{R}^{2}\right)$ provided

$$
\int_{\mathbb{B}_{1}} H^{\prime}(d(R)) \operatorname{det} \nabla \varphi_{n}(x) \mathrm{d} x \rightarrow \int_{\mathbb{B}_{1}} H^{\prime}(d(R)) \operatorname{det} \nabla \varphi(x) \mathrm{d} x
$$

whenever $\varphi_{n} \rightarrow \varphi$ in $W_{0}^{1,2}\left(\mathbb{B}_{1}, \mathbb{R}^{2}\right)$. This can be proved using the ideas of Coifman et al. [9].

Proposition 1. Let $u_{0}+\varphi \in \mathcal{A}^{-}$. Then

(a) $J\left(u_{0}, \varphi\right) \geq 0$ if $\varphi \in C_{0}^{\infty}\left(\mathbb{B}_{1}, \mathbb{R}^{2}\right)$, and

(b) $J\left(u_{0}, \varphi_{n}\right) \rightarrow J\left(u_{0}, \varphi\right)$ if $\varphi \in W_{0}^{1,2}\left(\mathbb{B}_{1}, \mathbb{R}^{2}\right)$ and the smooth maps $\varphi_{n} \rightarrow \varphi$ strongly in $W_{0}^{1,2}\left(\mathbb{B}_{1}, \mathbb{R}^{2}\right)$.

Proof. (a) Let $u_{0}+\varphi \in \mathcal{A}^{-}$, where $\varphi$ is smooth and has compact support in $\mathbb{B}_{1}$. We express $\varphi$ as

$$
\varphi=\left(\varphi_{1}, \varphi_{2}\right) \in W_{0}^{1,2}\left(\mathbb{B}_{1}, \mathbb{R}^{2}\right)
$$

Here

$$
\begin{aligned}
\varphi_{1}(R, \theta) & =a_{0}(R)+a_{1}(R) \cos \theta+b_{1}(R) \sin \theta+\sum_{n \geq 2}^{\infty}\left(a_{n}(R) \cos n \theta+b_{n}(R) \sin n \theta\right) \\
& =a_{1}(R) \cos \theta+b_{1}(R) \sin \theta+\eta_{1}(R, \theta), \\
\varphi_{2}(R, \theta) & =c_{0}(R)+c_{1}(R) \cos \theta+d_{1}(R) \sin \theta+\sum_{n \geq 2}^{\infty}\left(c_{n}(R) \cos n \theta+d_{n}(R) \sin n \theta\right) \\
& =c_{1}(R) \cos \theta+d_{1}(R) \sin \theta+\eta_{2}(R, \theta),
\end{aligned}
$$

and

$$
\int_{0}^{1} H^{\prime \prime}(d(R)) d^{\prime}(R)\left(a_{1}(R) d_{1}(R)-c_{1}(R) b_{1}(R)\right) \mathrm{d} R \leq 0 .
$$

For $\varphi \in C_{0}^{\infty}\left(\mathbb{B}_{1}, \mathbb{R}^{2}\right)$ we can write $J\left(u_{0}, \varphi\right)$ as

$$
\begin{aligned}
J\left(u_{0}, \varphi\right) & =\int_{\mathbb{B}_{1}} \frac{1}{2}|\nabla \varphi|^{2}+H^{\prime}\left(\operatorname{det} \nabla u_{0}\right) \operatorname{det} \nabla \varphi \mathrm{d} x \\
& =\int_{\mathbb{B}_{1}} \frac{1}{2}|\nabla \varphi|^{2}+H^{\prime}\left(\operatorname{det} \nabla u_{0}\right) \operatorname{det} \nabla \phi \mathrm{d} x
\end{aligned}
$$

where

$$
\begin{aligned}
\phi(R, \theta) & =\varphi(R, \theta)-\frac{1}{2 \pi} \int_{0}^{2 \pi} \varphi(R, \theta) \mathrm{d} \theta \\
& =\left(a_{1}(R) \cos \theta+b_{1}(R) \sin \theta+\eta_{1}(R, \theta), c_{1}(R) \cos \theta+d_{1}(R) \sin \theta+\eta_{2}(R, \theta)\right)
\end{aligned}
$$

Here we can replace $\varphi$ in $J\left(u_{0}, \varphi\right)$ with $\phi$ because of the equality

$$
\int_{0}^{2 \pi} \operatorname{det} \nabla \varphi(R, \theta) \mathrm{d} \theta=\int_{0}^{2 \pi} \operatorname{det} \nabla \phi(R, \theta) \mathrm{d} \theta .
$$


Letting $\eta=\left(\eta_{1}, \eta_{2}\right)$ we have in particular that

$$
\int_{0}^{1} \int_{0}^{2 \pi} \frac{\eta_{i}^{2}(R, \theta)}{R^{2}} R \mathrm{~d} R \mathrm{~d} \theta \leq \frac{1}{4} \int_{0}^{1} \int_{0}^{2 \pi} \frac{\eta_{i \theta}^{2}(R, \theta)}{R^{2}} R \mathrm{~d} R \mathrm{~d} \theta<\infty \quad i=1,2
$$

Here the coefficient $\frac{1}{4}$ in (3.25) comes from the fact that $\eta_{i}$ has nonvanishing Fourier modes of order two at least. Therefore

$$
\begin{aligned}
\int_{\mathbb{B}_{1}} H^{\prime}\left(\operatorname{det} \nabla u_{0}\right) \operatorname{det} \nabla \phi \mathrm{d} x & =\int_{\mathbb{B}_{1}} H^{\prime}\left(\operatorname{det} \nabla u_{0}\right) \operatorname{Det} \nabla \phi \mathrm{d} x \\
& =\int_{\mathbb{B}_{1}} H^{\prime}\left(\operatorname{det} \nabla u_{0}\right) \operatorname{div}\left(\phi_{1} \phi_{2 x_{2}},-\phi_{1} \phi_{2 x_{1}}\right) \mathrm{d} x \\
& =-\int_{\mathbb{B}_{1}} H^{\prime \prime}(d(R)) d^{\prime}(R)\left(\phi_{1} \phi_{2 x_{2}},-\phi_{1} \phi_{2 x_{1}}\right) \cdot \frac{x}{|x|} \mathrm{d} x \\
& =-\int_{\mathbb{B}_{1}} \frac{H^{\prime \prime}(d(R)) d^{\prime}(R) \phi_{1} \phi_{2 \theta}}{R} \mathrm{~d} x \\
& =-\pi \int_{0}^{1} H^{\prime \prime}(d(R)) d^{\prime}(R)\left(a_{1} d_{1}-c_{1} b_{1}\right) \mathrm{d} R \\
& -\int_{\mathbb{B}_{1}} \frac{H^{\prime \prime}(d(R)) d^{\prime}(R)}{R} \eta_{1} \eta_{2 \theta} \mathrm{d} x \\
& \geq-\int_{\mathbb{B}_{1}} \frac{H^{\prime \prime}(d(R)) d^{\prime}(R)}{R} \eta_{1} \eta_{2 \theta} \mathrm{d} x \\
& =-\int_{\mathbb{B}_{1}} H^{\prime \prime}(d(R)) d^{\prime}(R)\left(\eta_{1} \eta_{2 x_{2}},-\eta_{1} \eta_{2 x_{1}}\right) \cdot \frac{x}{|x|} \mathrm{d} x .
\end{aligned}
$$

Here we used integration by parts and the growth estimate of $H^{\prime}(d(R))$ near $R=0$, together with the fact that $\phi \in C_{0}^{\infty}$. In the last step we used the assumption (3.24).

By Lemmas 2 and 3,

$$
0 \leq g(R)=R H^{\prime \prime}(d(R)) d^{\prime}(R)<2 \text { for } R \in(0,1)
$$

From (3.25), (3.26) and (3.27) we conclude that

$$
\begin{aligned}
\int_{\mathbb{B}_{1}} H^{\prime}\left(\operatorname{det} \nabla u_{0}\right) \operatorname{det} \nabla \phi \mathrm{d} x & \geq-\int_{\mathbb{B}_{1}} H^{\prime \prime}(d(R)) d^{\prime}(R)\left(\eta_{1} \eta_{2 x_{2}},-\eta_{1} \eta_{2 x_{1}}\right) \cdot \frac{x}{|x|} \mathrm{d} x \\
& \geq-2 \int_{\mathbb{B}_{1}}\left|\frac{\eta_{1}}{R}\right|\left|\frac{\eta_{2 \theta}}{R}\right| \mathrm{d} x \\
& \geq-2\left(\int_{\mathbb{B}_{1}}\left|\frac{\eta_{1}}{R}\right|^{2} \mathrm{~d} x\right)^{\frac{1}{2}}\left(\int_{\mathbb{B}_{1}}\left|\frac{\eta_{2 \theta}}{R}\right|^{2} \mathrm{~d} x\right)^{\frac{1}{2}} \\
& \geq-\frac{2}{2}\left(\int_{\mathbb{B}_{1}}\left|\frac{\eta_{1 \theta}}{R}\right|^{2} \mathrm{~d} x\right)^{\frac{1}{2}}\left(\int_{\mathbb{B}_{1}}\left|\frac{\eta_{2 \theta}}{R}\right|^{2} \mathrm{~d} x\right)^{\frac{1}{2}} \\
& \geq-\frac{1}{2} \int_{\mathbb{B}_{1}}\left|\frac{\eta_{\theta}}{R}\right|^{2} \mathrm{~d} x .
\end{aligned}
$$


Thus for $\varphi \in C_{0}^{\infty}\left(\mathbb{B}_{1}, \mathbb{R}^{2}\right)$ satisfying (3.24) it follows that

$$
\begin{aligned}
J\left(u_{0}, \varphi\right) & =\int_{\mathbb{B}_{1}} \frac{1}{2}|\nabla \varphi|^{2}+H^{\prime}\left(\operatorname{det} \nabla u_{0}\right) \operatorname{det} \nabla \phi \mathrm{d} x \\
& =\int_{\mathbb{B}_{1}} \frac{1}{2}|\nabla \varphi|^{2}+H^{\prime}\left(\operatorname{det} \nabla u_{0}\right)\left(\left(\phi_{1} \phi_{2 x_{2}}\right)_{x_{1}}-\left(\phi_{1} \phi_{2 x_{1}}\right)_{x_{2}}\right) \mathrm{d} x \\
& =\int_{\mathbb{B}_{1}} \frac{1}{2}|\nabla \varphi|^{2}-\left(\phi_{1} \phi_{2 x_{2}},-\phi_{1} \phi_{2 x_{1}}\right) \cdot \frac{x}{|x|} H^{\prime \prime}(d(R)) d^{\prime}(R) \mathrm{d} x \\
& \geq \int_{\mathbb{B}_{1}} \frac{1}{2}|\nabla \varphi|^{2} \mathrm{~d} x-\frac{1}{2} \int_{\mathbb{B}_{1}}\left|\frac{\eta_{\theta}}{R}\right|^{2} \mathrm{~d} x \\
& \geq \int_{\mathbb{B}_{1}} \frac{1}{2}\left|\varphi_{R}\right|^{2} \mathrm{~d} x \\
& \geq 0
\end{aligned}
$$

proving part (a) of the proposition.

(b) Observe that $\operatorname{det} \nabla \varphi(x) \in \mathcal{H}^{1}\left(\mathbb{B}_{1}\right)$ (see e.g. [9] or [12]) and $H^{\prime}(d(R)) \in B M O\left(\mathbb{B}_{1}\right)$ (to be proved later). Suppose that $\varphi_{n} \in C_{0}^{\infty}\left(\mathbb{B}_{1}, \mathbb{R}^{2}\right)$ such that $\varphi_{n} \rightarrow \varphi$ strongly in $W_{0}^{1,2}\left(\mathbb{B}_{1}, \mathbb{R}^{2}\right)$. In particular, $\operatorname{det} \nabla \varphi_{n}(x) \rightarrow$ $\operatorname{det} \nabla \varphi(x)$ strongly in $\mathcal{H}^{1}\left(\mathbb{B}_{1}\right)$ (see [9]). Therefore $J\left(u_{0}, \varphi_{n}\right) \rightarrow J\left(u_{0}, \varphi\right)$ as $n \rightarrow \infty$. To finish the proof we show $H^{\prime}(d) \in B M O\left(\mathbb{B}_{1}\right)$. Recall that $d(R)$ is continuous on $[0,1]$ and positive away from the origin. Therefore $H^{\prime}(d)$ is bounded away from any fixed small disk around the origin. Fix $\epsilon>0$ small and choose $\delta>0$ such that

$$
\begin{aligned}
& \left(\frac{3}{2}-\epsilon\right) \ln R \leq H^{\prime}(d(R)) \leq\left(\frac{3}{2}+\epsilon\right) \ln R \\
& \left(\frac{3}{2}-\epsilon\right) \leq R H^{\prime \prime}(d(R)) d^{\prime} \leq\left(\frac{3}{2}+\epsilon\right) \quad \text { for } 0<R<\delta .
\end{aligned}
$$

We only need to show there exists a constant $A$ such that

$$
\frac{1}{|B|} \int_{B}\left|h-h_{B}\right| \mathrm{d} x \leq A
$$

for any disk $B$ which has nonempty intersection with $B_{\delta}(0)$. The proof of (3.31) follows from Poincaré's inequality and the estimate $\int_{B}|\nabla h| \mathrm{d} x \leq C \rho$ for any $B\left(x_{0}, \rho\right)$. The latter follows directly from (3.30).

Remark 1. The proof above shows that $J\left(u_{0}, \varphi\right) \geq 0$ for all $\varphi \in W_{0}^{1,2}\left(\mathbb{B}_{1}, \mathbb{R}^{2}\right)$ satisfying (3.24).

Proof of Theorem 1. It is clear from Proposition 1 that $u_{0}$ is a global minimizer of $I$ on $\mathcal{A}^{-}$. The uniqueness follows directly from the proof above. In fact, if

$$
I\left(u_{0}+\varphi\right)=I\left(u_{0}\right),
$$

then from (3.29) we have

$$
\int_{\mathbb{B}_{1}} \frac{1}{2}\left|\varphi_{R}\right|^{2} \mathrm{~d} x=0 .
$$


For $\varphi=\left(\varphi_{1}, \varphi_{2}\right)$ with

$$
\begin{aligned}
\varphi_{1}(R, \theta) & =a_{0}(R)+a_{1}(R) \cos \theta+b_{1}(R) \sin \theta+\sum_{n \geq 2}^{\infty}\left(a_{n}(R) \cos n \theta+b_{n}(R) \sin n \theta\right) \\
& =a_{0}(R)+a_{1}(R) \cos \theta+b_{1}(R) \sin \theta+\eta_{1}(R, \theta), \\
\varphi_{2}(R, \theta) & =c_{0}(R)+c_{1}(R) \cos \theta+d_{1}(R) \sin \theta+\sum_{n \geq 2}^{\infty}\left(c_{n}(R) \cos n \theta+d_{n}(R) \sin n \theta\right) \\
& =c_{0}(R)+c_{1}(R) \cos \theta+d_{1}(R) \sin \theta+\eta_{2}(R, \theta) .
\end{aligned}
$$

Rewriting (3.32) we have

$$
\int_{0}^{1}\left[\left(a_{0}^{\prime}(R)\right)^{2}+\left(c_{0}^{\prime}(R)\right)^{2}\right] R \mathrm{~d} R+\int_{0}^{1}\left[\sum_{i \geq 1}\left(\left(a_{i}^{\prime}(R)\right)^{2}+\left(b_{i}^{\prime}(R)\right)^{2}+\left(c_{i}^{\prime}(R)\right)^{2}+\left(d_{i}^{\prime}(R)\right)^{2}\right)\right] R \mathrm{~d} R=0
$$

Since $\varphi \in W_{0}^{1,2}\left(\mathbb{B}_{1}, \mathbb{R}^{2}\right)$, we have for $R \in(0,1)$

$$
\begin{aligned}
\int_{R}^{1} a_{0}^{2}(s) \mathrm{d} s & =\int_{R}^{1}\left(\int_{s}^{1} a_{0}^{\prime}(t) \mathrm{d} t\right)^{2} \mathrm{~d} s \\
& \leq \int_{R}^{1}\left(\int_{s}^{1}\left(a_{0}^{\prime}(t)\right)^{2} t \mathrm{~d} t \cdot \int_{s}^{1} \frac{1}{t} \mathrm{~d} t\right) \mathrm{d} s \\
& =0
\end{aligned}
$$

It then follows that $a_{0}(R)=0$ a.e. in $(0,1)$. Similar arguments show that $c_{0}(R)=0$ for a.e. $R \in(0,1)$, and when $i \geq 1$

A direct corollary of our theorem is the following:

$$
a_{i}(R)=b_{i}(R)=c_{i}(R)=d_{i}(R)=0 \text { for a.e. } R \in(0,1) .
$$

Corollary 1. The Bauman-Phillips-Owen map $u_{0}$ is the unique solution in $\mathcal{A}_{s}$ of the equilibrium equations.

Proof. As we pointed out in the introduction, any weak solution $u$ of the equilibrium equations in the class $\mathcal{A}_{s}$ shares the same properties as $u_{0}$. It then follows from the proof of Theorem 1 that $u$ is a global minimizer of $I$ in $\mathcal{A}^{-}$, and since the global minimizer is unique it must be that $u=u_{0}$.

\section{Final Remarks}

Below we state some open questions which are partly motivated by the work in this paper and partly by the review paper of Ball [4].

(1) Is $u_{0}$ stable with respect to small perturbations, and in particular those involving Fourier one modes in such a way as to violate (3.24)? Is it a global minimizer in the full class $\mathcal{A}_{g}$ defined in the introduction? Our result states that any mapping which lowers the energy must have Fourier one modes which violate condition (3.24). Some of our initial calculation (see the appendix) indicate that simple perturbations involving Fourier one modes violating (3.24) do not lower the energy.

(2) How smooth can we expect a global minimizer $u$ to be if $u_{0}$ itself is not a global minimizer? Is the global minimizer unique?

We point out that the maximal smoothness for a global minimizer under our boundary constraint is $C^{1}$. In fact, Bauman et al. [8] showed that any $C^{1, \alpha}$ solution to the equilibrium equation satisfies 
$\operatorname{det} \nabla u>0$ everywhere in the domain. This together with the fact that the map is two-to-one on the boundary immediately implies the following lemma (in discussion with J. Ball).

Lemma 4. If $u$ is a global minimizer in $\mathcal{A}$ with $u(1, \theta)=(\cos 2 \theta, \sin 2 \theta)$, then $u \notin C^{1, \beta}\left(\mathbb{B}_{1}, \mathbb{R}^{2}\right)$ for any $\beta>0$. Moreover, if $u$ is $C^{1}$ then $\operatorname{det} \nabla u$ vanishes in at least one point.

Proof. We claim that if $u$ is $C^{1}$ with the given boundary constraint then $\operatorname{det} \nabla u$ cannot be positive everywhere in $\mathbb{B}_{1}$. Otherwise, from degree theory, each point in $\mathbb{B}_{1}$ has two preimages and so $u$ is a local homeomorphism. In particular, by mapping each point to the other preimage, we can get a homeomorphism from $\mathbb{B}_{1}$ to $\mathbb{B}_{1}$ with no fixed point, which is impossible by Brouwer's fixed point theorem. Therefore we can not have $\operatorname{det} \nabla u(x)>0$ in $\mathbb{B}_{1}$. On the other hand, a global minimizer $u$ satisfies the equilibrium equation. If $u \in C^{1, \beta}$ for some $\beta>0$, by [7], we have $\operatorname{det} \nabla u(x)>0$ on $\mathbb{B}_{1}$, a contradiction. Hence $u \notin C^{1, \beta}\left(\mathbb{B}_{1}, \mathbb{R}^{2}\right)$ for any $\beta>0$. If a global minimizer $u$ is $C^{1}$, then finite energy forces $\operatorname{det} \nabla u \geq 0$ in $\mathbb{B}_{1}$. Since $\operatorname{det} \nabla u$ can not be positive everywhere, it vanishes in at least one point.

(3) If the boundary constraint is a one-to-one smooth mapping, can we determine the regularity and uniqueness of a global minimizer or of a weak solution to (2.3) and (2.4)?

The Bauman-Owen-Phillips example indicates that invertibility has an effect on regularity, but the double-covering boundary condition is clearly unsatisfactory from a physical point of view. It would be more interesting to determine the maximal smoothness for almost one-to-one minimizers or weak solutions to (2.3) subject to a smooth one-to-one boundary conditions.

\section{Appendix}

Here we consider a special class $\mathcal{C}$ of perturbations $u_{0}+\varphi$ of the Bauman-Owen-Phillips map $u_{0}$ with the property that

(A) there exist $u_{0}+\varphi \in \mathcal{C}$ such that $J\left(u_{0}, \varphi\right)<0$ (necessarily such $\varphi$ violate $(3.24)$ );

(B) no $u_{0}+\varphi \in \mathcal{C}$ decreases the energy of the map $u_{0}$, and in particular $I\left(u_{0}+\varphi\right)>I\left(u_{0}\right)$ whenever $\varphi \neq 0$. Assertions A and B are proved in Appendices A and B respectively. We have made a specific choice of the map $H$ for the calculations in Appendix B (see Prop. (2) below); Assertion A holds for any choice of $H$ consistent with the hypotheses described in Section 2. As before, let $d(R)=\operatorname{det} \nabla u_{0}$.

\section{Appendix A}

Let $\psi(R, \theta)=h(R)(-\sin \theta, \cos \theta)$, where $h$ is a 1-Lipschitz function to be chosen shortly, and consider

$$
\begin{aligned}
J\left(u_{0}, \psi\right) & =\int_{\mathbb{B}_{1}} \frac{1}{2}|\nabla \psi|^{2}+H^{\prime}(d) \operatorname{det} \nabla \psi \mathrm{d} x \\
& =2 \pi \int_{0}^{1}\left[\frac{1}{2}\left(\frac{h^{2}}{R^{2}}+{h^{\prime}}^{2}\right)+H^{\prime}(d) \frac{h h^{\prime}}{R}\right] R \mathrm{~d} R,
\end{aligned}
$$

which, if $h(0)=h(1)=0$, gives

$$
\frac{1}{2 \pi} J\left(u_{0}, \psi\right)=\frac{1}{2} \int_{0}^{1}\left[\frac{h^{2}}{R^{2}}+h^{\prime 2}-H^{\prime \prime}(d) d^{\prime} \frac{h^{2}}{R}\right] R \mathrm{~d} R .
$$

By part (ii) of Lemma 1 we can for any $\epsilon>0$ find $\delta>0$ such that

$$
\left|H^{\prime \prime}(d) d^{\prime}-\frac{3}{2 R}\right|<\frac{\epsilon}{R} \text { for all } 0<R<\delta .
$$


Hence

$$
\frac{1}{\pi} J\left(u_{0}, \psi\right) \leq \int_{0}^{1}\left[h^{\prime 2}-\left(\frac{1}{2}-\epsilon\right) \frac{h^{2}}{R^{2}}\right] R \mathrm{~d} R
$$

which on making the substitution $R=e^{s}$ and letting $f(s)=h\left(e^{s}\right)$, gives

$$
\frac{1}{2 \pi} J\left(u_{0}, \psi\right) \leq \int_{-\infty}^{0}\left[f^{\prime 2}-\left(\frac{1}{2}-\epsilon\right) f^{2}\right] \mathrm{d} s .
$$

With $\delta$ fixed and for any given $\lambda \geq 0$, we choose $f$ such that $\int_{-\infty}^{0} f^{\prime 2}(s) \mathrm{d} s=2$ while $\int_{-\infty}^{0} f^{2}(s) \mathrm{d} s \geq \lambda\left|\log \frac{\delta}{4}\right|$; for example, if $a:=\log \frac{\delta}{4}$ then one could take

$$
f(s)= \begin{cases}1+a-s & \text { if } a \leq s \leq a+1 \\ 1 & \text { if }(\lambda+1) a \leq s \leq a \\ s-((\lambda+1) a-1) & \text { if }(\lambda+1) a-1 \leq s \leq(\lambda+1) a \\ 0 & \text { otherwise }\end{cases}
$$

the choice of $a$ being such that $\operatorname{supp} \psi \subset B(0, \delta)$ and hence such that (5.2) above holds. It follows from (5.3) that $J\left(u_{0}, \psi\right)<0$ for this choice of $f$. Notice also that from $(5.1)$ we have that $J\left(u_{0}, k \psi\right)=k^{2} J\left(u_{0}, \psi\right)$ for any $k$. Since $\operatorname{det} \nabla u_{0}$ is continuous and strictly positive on the small annulus supp $\psi$ we can arrange that $\operatorname{det}\left(\nabla u_{0}+k \nabla \psi\right)>0$ on $\mathbb{B}_{1}$ for sufficiently small $k$; in particular $u_{0}+k \psi$ is an admissible variation with the property that $J\left(u_{0}, k \psi\right)<0$.

\section{Appendix B}

Let

$$
\gamma(P)=\left\{\begin{array}{cc}
\frac{1}{2}|P|^{2}+\frac{1}{\operatorname{det} P} & \operatorname{det} P>0 \\
+\infty & \text { otherwise }
\end{array}\right.
$$

The class $\mathcal{C}$ will consist of all perturbations $v \in \mathcal{A}_{g}$ of the form $v=u_{0}+\varphi$ where $\varphi(R, \theta)=h(R)(\cos \theta, \sin \theta)$ or $\varphi(R, \theta)=h(R)(-\sin \theta, \cos \theta)$. We claim that such maps cannot lower the energy.

Proposition 2. Let $H(s)=\frac{1}{s}$ if $s>0$ and $H(s)=+\infty$ otherwise. Suppose that $\operatorname{det} \nabla v>0$ almost everywhere, where $v=u_{0}+\varphi$ and $\varphi(R, \theta)=h(R)(\cos \theta, \sin \theta)$. Then if

$$
\int_{\mathbb{B}_{1}} H(\operatorname{det} \nabla v)-H(d(R)) \mathrm{d} x \geq \frac{1}{2} \int_{\mathbb{B}_{1}} H^{\prime}(d(R)) \operatorname{det} \nabla \varphi \mathrm{d} x,
$$

holds we have

$$
I\left(u_{0}+\varphi\right) \geq I\left(u_{0}\right)
$$

Proof. An easy calculation using (5.4) and the fact that $\int_{0}^{2 \pi} \nabla u_{0} \cdot \nabla \varphi \mathrm{d} \theta=0$ shows that

$$
I\left(u_{0}+\varphi\right)-I\left(u_{0}\right) \geq \frac{1}{2} \int_{\mathbb{B}_{1}}\left(|\nabla \varphi|^{2}+H^{\prime}(d(R)) \operatorname{det} \nabla \varphi\right) \mathrm{d} x
$$

(5.5) shows that, owing to the special form of the perturbation, the difference in energies is bounded below by a different functional to $J$ : the subtle difference is the position of the factor $\frac{1}{2}$, and this, by the analysis leading to $(3.28)$ leads to the inequality

proving the proposition.

$$
I\left(u_{0}+\varphi\right)-I\left(u_{0}\right) \geq \frac{1}{2} \int_{\mathbb{B}_{1}}\left|\varphi_{R}\right|^{2} \mathrm{~d} x
$$


In order to show that (5.4) holds it is clearly sufficient to show

$$
\int_{0}^{2 \pi} \frac{1}{\operatorname{det} \nabla v}-\frac{1}{\operatorname{det} \nabla u_{0}} \mathrm{~d} \theta \geq-\frac{1}{2} \int_{0}^{2 \pi} \frac{\operatorname{det} \nabla\left(v-u_{0}\right)}{\operatorname{det}^{2} \nabla u_{0}} \mathrm{~d} \theta
$$

since (5.4) then follows by integration in $R$. In the following we write

$$
\begin{aligned}
\operatorname{det}\left(\nabla u_{0}+\nabla \varphi\right) & =\frac{2 r r^{\prime}}{R}+\frac{h h^{\prime}(R)}{R}+\left(\frac{r^{\prime} h}{R}+\frac{2 r h^{\prime}}{R}\right) \sin \theta \\
& =d+f+g \sin \theta
\end{aligned}
$$

To prove (5.6) we consider several cases based on the range of $f(R)$. Unless stated otherwise, all integrations are with respect to $\theta$.

Case (i): $f(R)<0$ or $f(R) \geq d(R)$.

By Jensen's inequality we have

$$
\begin{aligned}
\int_{0}^{2 \pi} \frac{1}{\operatorname{det}(\nabla u+\nabla \varphi)} & \geq \frac{(2 \pi)^{2}}{\int_{0}^{2 \pi} \operatorname{det}(\nabla u+\nabla \varphi)} \\
& =\int_{0}^{2 \pi} \frac{1}{d+f} \\
& =\int_{0}^{2 \pi} \frac{1}{d}-\int_{0}^{2 \pi} \frac{f}{d(d+f)} \\
& \geq \int_{0}^{2 \pi} \frac{1}{d}-\frac{1}{2} \int_{0}^{2 \pi} \frac{f}{d^{2}}
\end{aligned}
$$

which is (5.6).

Case (ii): $0<f(R)<d(R)$.

$$
\begin{aligned}
I(R):= & \int_{0}^{2 \pi} \frac{1}{\operatorname{det}(\nabla u+\nabla \varphi)}-\int_{0}^{2 \pi} \frac{1}{\operatorname{det} \nabla u}+\frac{1}{2} \int_{0}^{2 \pi} \frac{\operatorname{det} \nabla \varphi}{\operatorname{det}^{2} \nabla u} \\
& =\int_{0}^{2 \pi} \frac{1}{d+f+g \sin \theta} \mathrm{d} \theta-2 \pi \frac{2 d-f}{2 d^{2}}
\end{aligned}
$$

and

$$
\int_{0}^{2 \pi} \frac{1}{d+f+g \sin \theta} \mathrm{d} \theta=\frac{2 \pi}{\sqrt{(d+f)^{2}-g^{2}}}
$$

by elementary computation. The integrals $\int_{0}^{2 \pi} \frac{1}{d+f \pm g \sin \theta} \mathrm{d} \theta$ are well-defined for almost every $R \in(0,1)$ because $\operatorname{det} \nabla v>0$ a.e. by assumption. Since

$$
\frac{2 \pi}{\sqrt{(d+f)^{2}-g^{2}}} \geq \frac{2 \pi}{d-f}
$$


the inequality follows from

$$
\begin{aligned}
g^{2} & =\left(\frac{r^{\prime} h}{R}+\frac{2 r h^{\prime}}{R}\right)^{2} \\
& \geq 4 \cdot 2 \frac{r r^{\prime}}{R} \frac{h h^{\prime}}{R} \\
& =4 d f .
\end{aligned}
$$

Therefore

$$
\begin{aligned}
I(R) & \geqslant 2 \pi \cdot\left(\frac{1}{d-f}-\frac{2 d-f}{2 \mathrm{~d}^{2}}\right) \\
& =2 \pi \cdot \frac{2 \mathrm{~d}^{2}-(d-f)(2 d-f)}{(d-f) \mathrm{d}^{2}} \\
& \geq 0 .
\end{aligned}
$$

A similar analysis holds for $\varphi(R, \theta)=h(R)(\sin \theta, \cos \theta)$. The cases $\varphi(R, \theta)=h(R)(-\sin \theta, \cos \theta)$ and $\varphi(R, \theta)=$ $h(R)(-\cos \theta, \sin \theta)$ then follow automatically. We also note that the argument above easily extends to comparison functions of the form

$$
v(R, \theta)=u_{0}(R, \theta)+h(R)\left(\cos \left(\theta+\theta_{0}(R)\right), \sin \left(\theta+\theta_{0}(R)\right)\right)
$$

for any $\theta_{0}(R)$. In fact, we have

$$
\operatorname{det} \nabla v=\frac{2 r r^{\prime}}{R}+\frac{h h^{\prime}}{R}+g \sin \left(\theta+\varphi_{0}(R)\right)
$$

with

$$
g^{2}(R)=\frac{\left(2 r h^{\prime}+r^{\prime} h\right)^{2}+\left(2 r h \theta_{0}^{\prime}\right)^{2}}{R^{2}} \geq 4 d f .
$$

From (5.7) and (5.8) we can easily repeat the proof above and conclude that (5.4) holds for such comparison functions. In particular this implies that functions of the form

$$
v(R, \theta)=u_{0}(R, \theta)+(a(R) \cos \theta-b(R) \sin \theta, b(R) \cos \theta+a(R) \sin \theta)
$$

cannot lower the energy.

\section{REFERENCES}

[1] E. Acerbi and N. Fusco, Semicontinuity problems in the calculus of variations. Arch. Rational Mech. Anal. 86 (1984) $125-145$.

[2] J.M. Ball, Convexity conditions and existence theorems in nonlinear elasticity. Arch. Rational Mech. Anal. 63 (1977) $337-403$.

[3] J.M. Ball, Discontinuous equilibrium solutions and cavitation in nonlinear elasticity. Phil. Trans. Roy. Soc. London A 306 (1982) 557-611.

[4] J.M. Ball, Some open questions in elasticity. Geometry, mechanics, and dynamics. Springer, New York (2002) 3-59.

[5] J.M. Ball, J. Currie and P. Olver, Null Lagrangians, weak continuity and variational problems of arbitrary order. J. Func. Anal. 41 (1981) 135-174.

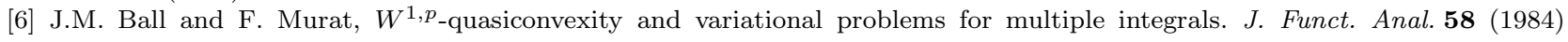
$225-253$.

[7] P. Bauman, N. Owen and D. Phillips, Maximum Principles and a priori estimates for a class of problems in nonlinear elasticity. Ann. Inst. H. Poincaré Anal. non Linéaire 8 (1991) 119-157.

[8] P. Bauman, N. Owen and D. Phillips, Maximal smoothness of solutions to certain Euler-Lagrange equations from noninear elasticity. Proc. Roy. Soc. Edinburgh 119A (1991) 241-263. 
[9] R. Coifman, P. L. Lions, Y. Meyer and S. Semmes, Compensated compactness and hardy spaces. J. Math. Pures. Appl. 72 (1993) $247-286$.

[10] C. Horgan and D. Polignone, Cavitation in nonlinearly elastic solids; A review. Appl. Mech. Rev. 48 (1995) 471-485.

[11] R. Knops and C. Stuart, Quasiconvexity and uniqueness of equilibrium solutions in nonlinear elasticity. Arch. Rational Mech. Anal. 86 (1984) 233-249.

[12] S. Müller, Higher integrability of determinants and weak convergence in $L^{1}$. J. reine angew. Math. 412 (1990) $20-34$.

[13] R. Ogden, Large-deformation isotropic elasticity: on the correlation of theory and experiment for compressible rubberlike solids. Proc. Roy. Soc. Edinburgh 328A (1972) 567-583.

[14] P. Quintela-Estevez, Critical point in the energy of hyperelastic materials. RAIRO: Math. Modél. Numér. Anal. 25 (1990) $103-132$.

[15] J. Sivaloganathan, The generalized Hamilton-Jacobi inequality and the stability of equilibria in nonlinear elasticity. Arch. Rational Mech. Anal. 107 (1989) 347-369.

[16] J. Sivaloganathan, Singular minimizers in the calculus of variations: a degenerate form of cavitation. Ann. Inst. H. Poincaré Anal. non linéaire 9 (1992) 657-681.

[17] J. Sivaloganathan, On the stability of cavitating equilibria. Q. Appl. Math. 53 (1995) 301-313.

[18] J. Spector, Linear deformations as global minimizers in nonlinear elasticity. Q. Appl. Math. 52 (1994) 59-64.

[19] V. Šverák, Regularity properties of deformations with finite energy. Arch. Rational Mech. Anal. 100 (1988) 105-127.

[20] A. Taheri, Quasiconvexity and uniqueness of stationary points in the multi-dimensional calculus of variations. Proc. Amer. Math. Soc. 131 (2003) 3101-3107.

[21] K. Zhang, Polyconvexity and stability of equilibria in nonlinear elasticity. Quart. J. Mech. appl. Math. 43 (1990) $215-221$.

[22] K. Zhang, Energy minimizers in nonlinear elastostatics and the implicit function theorem. Arch. Rational Mech. Anal. 114 (1991) 95-117. 